\title{
Dependence of the drag over Super Hydrophobic and Liquid Infused Surfaces on the textured surface and Weber number.
}

\author{
Edgardo J. García Cartagena · Isnardo \\ Arenas · Matteo Bernardini · Stefano \\ Leonardi
}

Received: date / Accepted: date

\begin{abstract}
Direct Numerical Simulations of a turbulent channel flow have been performed. The lower wall of the channel is made of staggered cubes with a second fluid locked in the cavities. Two viscosity ratios have been considered, $m=\mu_{1} / \mu_{2}=0.02$ and 0.4 (the subscript 1 indicates the fluid in the cavity and 2 the overlying fluid) representing superhydrophobic surfaces (SHS) and liquid infused surfaces (LIS) respectively. A first set of simulations with a slippery interface has been performed and results agree well with those in literature for perfect slip conditions and Stokes approximations.

To assess how the dynamics of the interface affects the drag, a second set of DNS has been carried out at $W e=100$ and 1,000 corresponding to $W e^{+} \simeq 10^{-3}$ and $10^{-2}$. The deformation of the interface is fully coupled to the Navier-Stokes equation and tracked in time using a Level Set Method. Two gas fractions, $G F=0.5$ and 0.875 , have been considered to assess how the spacing between the cubes affects the deformation of the interface and therefore the drag. For the dimensions of the substrate here considered, under the ideal assumption of flat interface, staggered cubes with $G F=0.875$ provide about $20 \%$ drag reduction. However, a rapid degradation of the performances is observed when the dynamics of the interface is considered, and the same
\end{abstract}

This work was supported under ONR MURI Grants N00014-12-0875 and N00014-12- 10962, Program Manager Dr. Ki-Han Kim. Numerical simulations were performed on the Texas Advanced Computer Center.

Edgardo J. García Cartagena

The University of Texas at Dallas

Isnardo Arenas

The University of Texas at Dallas

Matteo Bernardini

Universita' di Roma "La Sapienza"

Stefano Leonardi

The University of Texas at Dallas

E-mail: stefano.leonardi@utdallas.edu 
geometry increases the drag of about $40 \%$ with respect to a smooth wall. On the other hand, the detrimental effect of the dynamics of the interface is much weaker for $G F=0.5$.

Keywords Drag reduction · Liquid Infused Surfaces · Super-Hydrophobic surfaces · DNS · Level Set

\section{Introduction}

Theoretical studies and numerical simulations have proved that Superhydrophobic Surfaces (SHS) and Liquid Infused Surfaces (LIS) reduce the drag with respect to a smooth wall. SHS and LIS consist of a textured surface that traps either air or an oil which sustains a slip velocity and reduces the wall shear stress. In general, SHS should reduce more the drag because the viscosity of the fluid in the substrate is much smaller than that of oil. On the other hand, LIS have been shown to be more resistant to pressure fluctuations [1], and still have potential to reduce the drag up to $35 \%$ [2] in turbulent flow. For increased robustness of LISs patterned wettability have been proposed to prevent drainage of the infused liquid [3]. It has been proven that this friction reduction is kept as long as the interface remains flat without failure.

SHSs have been studied extensively by numerical simulations. Given the high value of the viscosity of water compared to that of air, for continuity of the shear stress at the interface, the velocity gradient on the air-water interface is expected to be very small. Based on this, in most of the numerical studies SHS have been modeled as alternating regions of no-slip and free-slip boundary conditions without solving the flow in the texture $([4-7])$.

The assumption of perfect slip cannot be made in case of LIS because the viscosity of the lubricant is of the same magnitude order of that of the main stream and then the shear at the interface is not zero. Fu et al. [8] performed DNS of two superposed fluids in a turbulent channel with one textured wall made of either longitudinal or square bars for a wide range of viscosity ratio, from SHS to LIS. The interface between the two fluids was forced to be horizontal and slippery in the streamwise and spanwise direction. Results agreed well with the model of Schönecker et al. [9] to determine the slip length for a given gas fraction of the substrate. They also demonstrated that correlation between the amount of drag reduction and the slip length in wall units developed by Rastegari and Akhavan [10] can be extended to LIS. Therefore, the drag reduction achieved using LIS can be described using the framework established for superhydrophobic surfaces.

Under the ideal condition of a flat interface, the drag is reduced by both LIS and SHS. However, failure is observed in experiments due to drainage of the air/oil from the cavities and the consequent loss of the Cassie state [1, 11,12]. Golovin et al. [13] explained that in real applications the stagnation pressure will expose asperities or produce meniscus curvature that ultimately will increase drag in SHSs. 
Although the robustness and stability of the interface is clearly of great importance, all numerical simulations in literature, to our knowledge, assume a flat interface between the two fluids, while linear analysis of Hopper and Boyd [14] and Hinch [15] has shown that a discontinuity of viscosity between the two fluids induces a flow instability and deformations of the interface.

The deformation of the interface has been studied by Seo et al. [16]. They imposed alternating free-slip and no-slip boundary condition on a horizontal wall to mimic an array of aligned cubes. The calculated pressure field was used as forcing to calculate the deformation of the interface. They attributed the failure of the interface to an increased pressure fluctuation that develop at the leading edge of posts textures in correspondence of the stagnation points. A relationship of texture size with surface tension was proposed by the authors to predict potential rupture of the air-water interface and an upper bound on the texture size is suggested for robust operation of SHSs. This limit is only an upper bound because the dynamics of the interface is not coupled to the flow field, and the pressure in a real scenario could be larger.

In the present paper we further corroborate their findings by coupling the dynamics of the interface with the solution of the Navier-Stokes equations. Both fluids are solved and the texture is modeled with an immersed boundary method. The objective of the paper is to quantify the deformation of the interface and how it affects the drag for two different gas fraction $G F=0.5$ and $G F=0.875$, varying the Weber number.

\section{Numerical procedure}

We consider the motion of two superposed, incompressible and immiscible fluids in a turbulent channel with a textured surface of staggered cubes. By using the channel-half height $h$ and the bulk velocity $U_{b}$ as length and velocity scales, density and viscosity of fluid 2 (main stream) as reference quantities, the Navier Stokes and continuity equations can be written in dimensionless form as

$$
\begin{aligned}
& \frac{\partial U_{i}}{\partial t}+\frac{\partial U_{i} U_{j}}{\partial x_{j}}=-\frac{\partial P}{\partial x_{i}}+\frac{1}{R e} \frac{\partial}{\partial x_{j}}\left[2 \tilde{\mu}(\phi) S_{i j}\right]+\Pi \delta_{i 1}+\frac{1}{W e} \kappa n_{i} \delta(\phi) \\
& \frac{\partial U_{i}}{\partial x_{i}}=0
\end{aligned}
$$

where $U_{i}$ are the velocity components in the streamwise $(i=1)$, wall-normal $(i=2)$ and spanwise direction $(i=3), \Pi$ is the pressure gradient required to maintain a constant flow rate, $P$ is the pressure, $n$ the normal vector to the interface, $\phi$ is the signed distance function from the interface, $\delta$ is the Dirac delta function at the interface $(\phi=0), \kappa$ is the curvature, and $S_{i j}$ is the symmetric part of the velocity gradient tensor. The function $\phi$ is used to mark the two fluids and it also appears in the non-dimensional factor $\tilde{\mu}=m+(1-$ $m) H$, where $m=\mu_{1} / \mu_{2}$ is the viscosity ratio and $H$ the Heaviside function, 
which is $H=0$, when $\phi<0$ (fluid 1, in the substrate), and $H=1$ when $\phi>0$ (fluid 2). The two fluids are assumed to have the same density. The Reynolds number is $R e=U_{b} h / \nu_{2}=2,800$ where $\nu_{2}$ is the kinematic viscosity of the overlying flow and it corresponds to a friction Reynolds number $R e_{\tau}=u_{\tau} h / \nu_{2}=180$ for a smooth channel where $u_{\tau}$ is the friction velocity. The Weber number is $W e=\rho U_{b}^{2} h / \sigma, \sigma$ being the surface tension.

The Navier Stokes equations have been discretized in a Cartesian coordinate system using a staggered configuration for the velocity components and second order finite difference approximations. The discretized system is advanced in time by a third order Runge-Kutta algorithm coupled with the Crank-Nicolson scheme combined with the fractional step method where viscous terms are treated implicitly and the convective terms explicitly. The large sparse matrix resulting form the implicit terms is inverted by an approximate factorization technique. At each time step the momentum equations are advanced with the pressure of the previous step, obtaining an intermediate nonsolenoidal velocity field. Then the Poisson equation is solved to enforced the solenoidal constraint, through Fourier transform method. The numerical details can be found in Orlandi [17]. The roughness is modeled by the efficient immersed boundary technique described in detailed by Orlandi \& Leonardi [18].

The tracking of the interface is achieved by solving the level set equation

$$
\frac{\partial \phi}{\partial t}+\frac{\partial\left(U_{i} \phi\right)}{\partial x_{i}}=0
$$

which is written in conservative form due to flow incompressibility. The numerical discretization of equation 2 presents challenges related to the sharp variation of viscosity $\tilde{\mu}(\phi)$ across the interface as well as the presence of the Dirac delta function for the application of the force due to surface tension. This problem is alleviated by giving a fixed thickness $\epsilon$ to the interface [19], proportional to the mesh spacing and introducing accordingly a smeared Heaviside function $\left(H_{\epsilon}\right)$ and mollified delta function $\left(\delta_{\epsilon}\right)$. Note that while this spreading of the interface is essential to avoid the onset of numerical oscillations it should be chosen as small as possible ( 2 points on both sides are a common value) to guarantee the representation of a "sharp" interface on the mesh. The level set equation is solved using a high-order accurate approximations for the convective term to avoid un-physical oscillations. A weighted essentially non oscillatory (WENO) reconstruction is used, which provides third order accuracy for the spatial derivatives [20]. The level set equation is advanced in time using the same third-order Runge Kutta algorithm implemented to solve the Navier Stokes equations.

Equation 2 will move correctly the zero level set, however it does not guarantee that $\phi$ will remain a distance function. For this purpose a re-initialization method is performed at each time step. This procedure is carried out by following the method developed by [21], which guarantees to conserve the mass of each fluid with high accuracy. To apply the surface tension, the continuous surface force (CSF) method is used, which adds the interfacial tension 
(a)
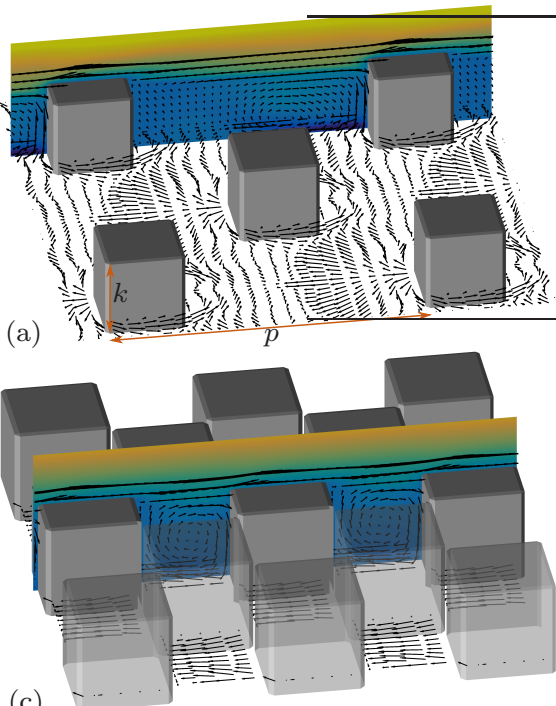

(b)
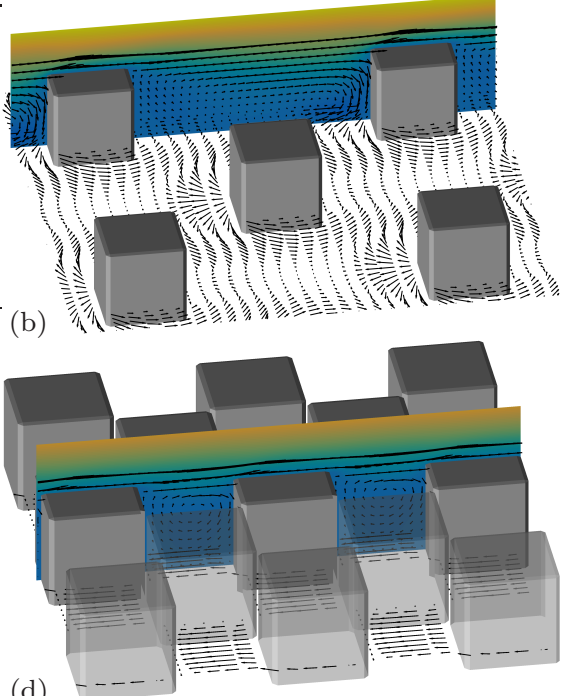

Fig. 1 Time averaged velocity vectors superposed to color contours of streamwise velocity: $W e=0:$ (a) $G F=0.875, m=0.02$, (b) $G F=0.875 m=0.4$, (c) $G F=0.5, m=0.02$, (d) $G F=0.5, m=0.4$

as a body force to the discretized Navier Stokes equation. For an accurate evaluation of the curvature the PROST method [22] is used in our level set formulation.

\section{Flow Configuration}

Direct numerical simulations of two superposed fluids in a channel with a textured surface made of staggered cubes have been performed. The height of the cubes in the substrate is $k=0.05 h$. Two gas fractions (GF) are considered with pitch $p / k=2$ for a $G F=0.5$ and $p / k=4$ for a $G F=0.875$ (Fig.1). The gas fraction is defined as the ratio of fluid interface area to the total area of the surface. Two viscosity ratios are considered $m=\mu_{1} / \mu_{2}=0.02$ and 0.4 , to mimic SHSs (water over air) and LISs and (water over heptane) respectively. A first set of simulations, with an ideal slippery interface, undeformable, at $W e=0$ has been performed. In order to study more realistic cases with a finite surface tension, two sets of simulations have been carried out for $W e=100$ and 1,000, corresponding in viscous units to a $W e^{+} \simeq \mu_{2} u_{\tau} / \sigma \simeq 10^{-3}$ and $10^{-2}$ respectively. Note that given the Reynolds and Weber numbers here investigated, assuming a water-air surface tension of $0.07 \mathrm{~N} / \mathrm{m}$, kinematic viscosity of water $10^{-6} \mathrm{~m}^{3} / \mathrm{s}$ and water density of $10^{3} \mathrm{~kg} / \mathrm{m}^{3}$, the the flow configurations simulated in this work can be achieved experimentally only in microchannels. Results are compared with those obtained with a flat interface (at $W e=0$ ) to highlight the detrimental effect 
of the deformation of the interface to the drag reduction. We point out that in our approach the interface between the two fluids is assumed to be effectively pinned at the post edges. As discussed by Seo et al. [16], this assumption can be interpreted as an asymptotic model for a contact line moving on a round corner whose curvature is much smaller than the post size and with a microscopic contact angle that is always between the nominal advancing and receding contact angles. We also highlight that the goal of the present work is to investigate the effect of the surface tension on turbulent drag reduction and no attempts have been made to study the conditions for the interface failure, with the subsequent transition from the Cassie-Baxter to the Wenzel state.

The computational box is $6.4 h \times 2.05 h \times 3.2 h$ in the streamwise $\left(x_{1}\right)$, wallnormal $\left(x_{2}\right)$ and spanwise $\left(x_{3}\right)$ directions respectively. The additional $0.05 h$ increase in channel height corresponds to the cavity height of the textured surface with plane of the crest at $y / h=-1$. Periodic boundary conditions are applied in the horizontal direction and no-slip condition is imposed at the walls. The computational grid is $1280 \times 384 \times 640$ in the streamwise, wallnormal and spanwise directions respectively. A non-uniform grid is used in the wall normal direction with 40 points clustered within the textured substrate.

All the simulations are carried out imposing a constant flow rate.

\section{Ideal case, flat interface}

Time averaged velocity vectors in horizontal and vertical planes are shown in Fig. 1. For a gas fraction $G F=0.5$, the momentum near the side walls of the cavities is very small, and the flow structure is mainly two-dimensional with a re-circulation in the vertical plane between two consecutive cubes, similar to that occurring over squared cavities. For the larger gas fraction, the flow in the textured surface becomes three-dimensional, resembling more closely the wake behind a wall mounted isolated cube. On the horizontal plane, two separated regions are observed behind the cubes, with opposite circulation. Two streams from the sides of the cube merge downstream the separated regions and in correspondence, an ejection of the flow is observed. The upward flow is redirected in the streamwise direction by the main stream and a recirculation is formed upstream of the next cube. The length of this separated region in the vertical plane is much larger than that relative to $G F=0.5$. Comparing the two set of simulations at different viscosity ratio, in general a similar flow structure is observed in the substrate, however, the intensity of the velocity is larger for SHS (due to the smaller viscosity in the substrate, $m=0.02$ ). Near the leading edge of the cubes velocity vectors are tilted upward. This tendency to ejections at the leading edge of the cube will be addressed further in the next section as it is expected to promote a deformation of the interface (although for $W e=0$ the surface tension is large enough to prevent any deformation of the interface). 

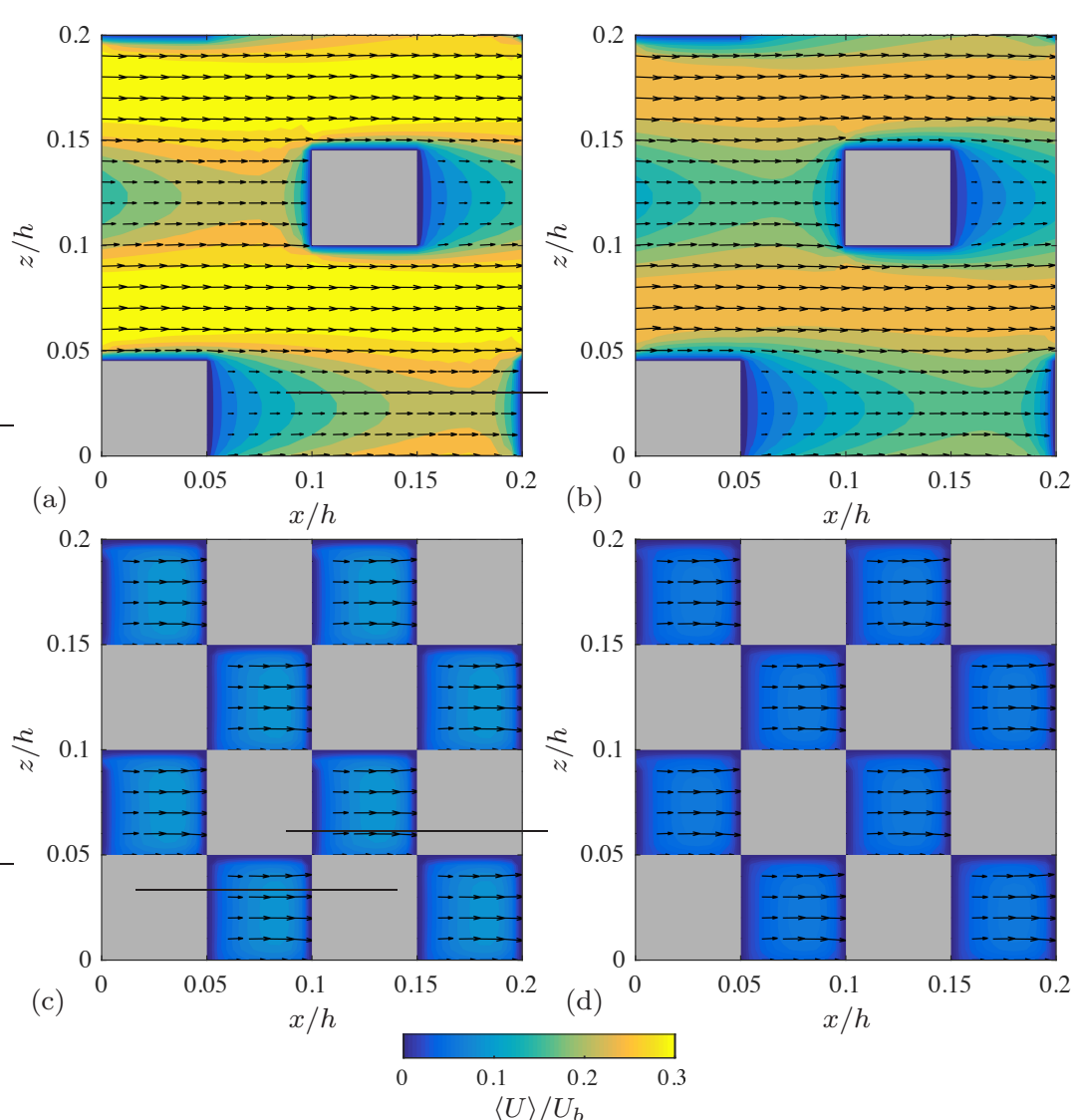

Fig. 2 Time averaged streamwise velocity on the horizontal plane at the crest plane for $W e=0$ : (a) with gas fraction $G F=0.875$, viscosity ratio $m=0.02$, (b) $G F=0.875$, $m=0.4$, (c) $G F=0.5, m=0.02$, (d) $G F=0.5, m=0.4$.

The slip velocity $\left(U_{s}\right)$, that is the time averaged velocity at the interface between the two fluids, is highly anisotropic for the case with sparse cubes $(G F=0.875)$ as shown in Fig.2. Slippage is large in the longitudinal streaks with no cubes and much smaller between two consecutive cubes aligned in the streamwise direction. In the limit of negligible viscosity in the textured surface, Ybert et al. [23] developed a model correlating the slip length $(b)$ and the gas fraction for an array of aligned cubes

$$
b / p=0.325 /\left[(1-G F)^{1 / 2}\right]-0.44,
$$

where the slip length can be defined as $b=U_{s} / \partial \bar{U}_{i} /\left.\partial n\right|_{y=-h},[24,25]$ representing the distance at which the mean velocity would be zero extrapolating into the surface the mean velocity gradient at the interface $\partial \bar{U}_{i} /\left.\partial n\right|_{y=-h}$. While in Ybert et al. [23] the cubes are aligned with the flow direction and equally spaced in streamwise and spanwise direction, here each row is shifted half wavelength in the spanwise direction with respect to the previous one. 


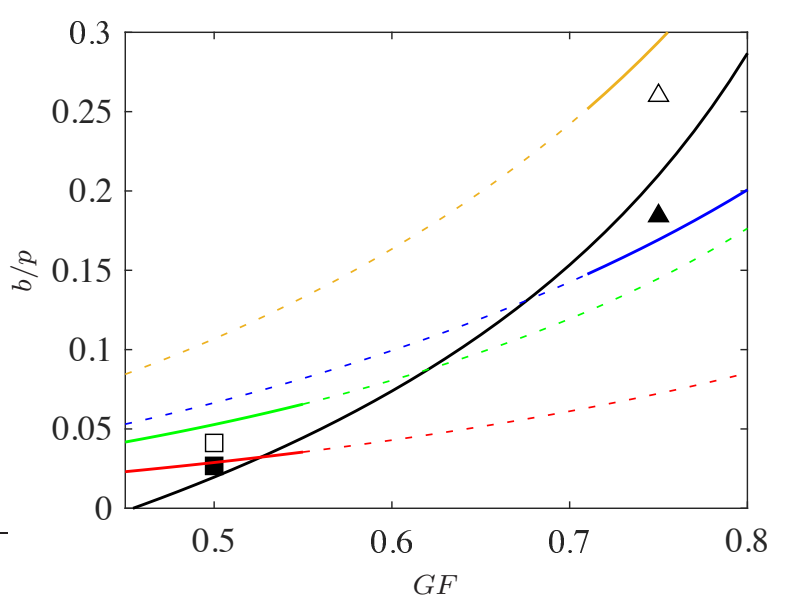

Fig. 3 Normalized slip length $b / p$ as a function of gas fraction: $(\square) G F=0.5 ;(\triangle) G F=$ 0.875 , empty symbols $m=0.02$, filled $m=0.4$. (—) eq.3 from Ybert et al [23]. The model from Schönecker et al. [9] is also included as reference: (-) and (-) for transversal bars with $m=0.4$ and 0.02 respectively; (-) and ( $(-)$ for longitudinal bars with $m=0.4$ and $m=0.02$ respectively.

Considering the flow pattern in Fig. 2, and the predominance of the high speed streaks on the overall slip velocity, the present configuration would have a similar flow structure of an aligned array of cubes with a pitch equal to $\tilde{p}=2 k$. The gas fraction of the equivalent array of aligned cubes would be $G F=1-(k / \tilde{p})^{2}=0.75$. Using $\tilde{p}=2 k$, and $G F=0.75$ the results with $m=0.02$ compare well with eq.3 as shown in Fig. 3 . The value of the slip length is slightly larger than that predicted by the model. In fact, while the slip velocity in the high speed streak should be basically the same for both configurations (aligned cubes and staggered cubes), the spacing and velocity between two streamwise aligned cubes is larger in our layout with respect to that considered by Ybert et al [23]. Given the predominance of the high speed streaks observed in Figure 2, results are also compared with the model proposed by [9], which incorporates the effects of viscosity of the fluid within the cavity when exposed to Stokes flow for longitudinal and square bars:

$$
b=\frac{p \ln \left(\sec \left(a \frac{\pi}{2}\right)\right)}{c \pi+\frac{m}{2 a D} \ln \left(\frac{1+\sin \left(\frac{\pi a}{2}\right)}{1-\sin \left(\frac{\pi a}{2}\right)}\right)}
$$

where $D$ is a geometric parameter related to the maximum local slip length, $m$ is the dynamic viscosity ratio between the external fluid and lubricant (i.e. $\left.m=\mu_{l u b} / \mu_{\text {ext }}\right)$, and $c=1$ and 2 for streamwise and transverse grooves, respectively. Using the modeling derived by [9], given the area fraction $a, D$ can be calculated for a given surface morphology. In particular for transverse grooves $D \approx 0.123$.

Present numerical results, normalized by the equivalent pitch $(p=2 k)$ agree well with equation 4 as shown in Fig. 3 and seem to indicate that arrays 


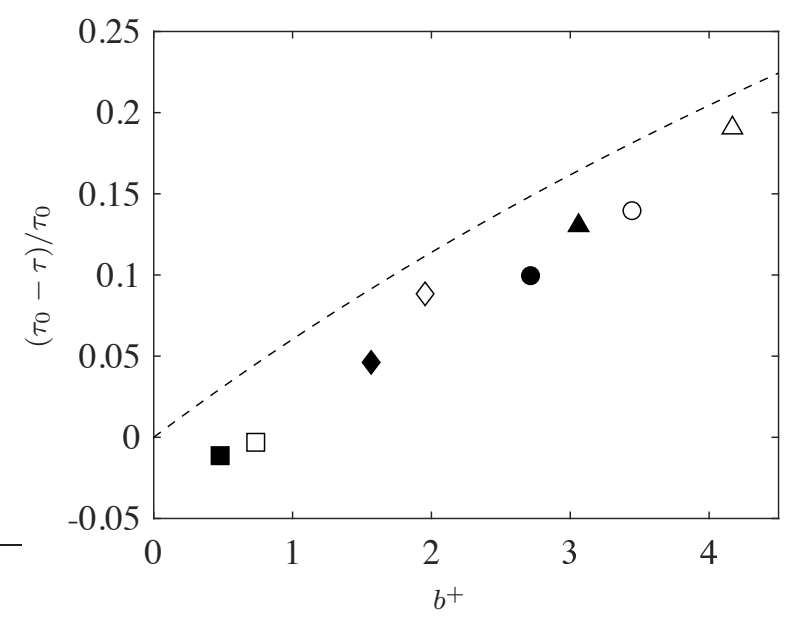

Fig. 4 Drag reduction as function of slip length $b^{+}$in wall units: staggered cubes $G F=$ $0.5, m=0.02(\square)$; staggered cubes $G F=0.5, m=0.4(\mathbf{\square})$; staggered cubes $G F=0.875$, $m=0.02(\triangle)$; staggered cubes $G F=0.875, m=0.4(\mathbf{\Delta})$; longitudinal bars from [8], $G F=$ 0.5: $k=0.05 h$ and $m=0.1(\diamond) ; k=0.05 h$ and $m=0.4(\diamond) ; k=0.1 h$ and $m=0.1(\bigcirc)$; $k=0.1 h$ and $m=0.4(\mathbf{O}) ;(--)$ analytic results of Rastegari and Akhavan [10].

of staggered cubes with small gas fraction behave as transversal rods, where the streamwise spacing is the dominant length scale. When the gas fraction is much larger and the spanwise spacing allows the formation of streaks without obstacles perpendicular to the main stream, the slip length is similar to that of longitudinal bars with an equivalent pitch equal to the periodicity of the longitudinal alley of high speed fluid.

Drag reduction $\left(D R=\left(\tau_{0}-\tau\right) / \tau_{0}\right.$, where $\tau_{0}$ is the wall shear stress of a smooth channel and $\tau$ is the shear stress of either SHS or LIS) as function of slip length $b^{+}$in wall units is shown in Fig.4. Results are in good agreement with the correlation proposed by Rastegari and Akhavan [10]

$$
D R=b^{+} /\left[b^{+}+\left(R e / R e_{\tau}\right)\right]
$$

The higher is the gas fraction, the larger is the amount of drag reduction. The maximum DR, for the value of $p^{+}$here considered is of the order of $20 \%$ for staggered cubes with $G F=0.875$. For a given substrate, SHS presents a smaller drag than LIS. Regardless of the different viscosity ratio and geometry of the textured surface, the qualitative agreement with eq. 5 indicates that the mechanism leading to drag reduction is to a good approximation dominated by the overall slippage and reduction of the shear for both SHS and LIS. With respect to eq. 5 , which assumes a shear-free boundary condition over the gas/liquid region, present simulations account for the frictional and pressure drag taking place in the underlying fluid. It is therefore expected that the amount of drag reduction is lower, the difference corresponding to the drag below the crests plane. Moreover, eq. 5 does not take into account the presence 


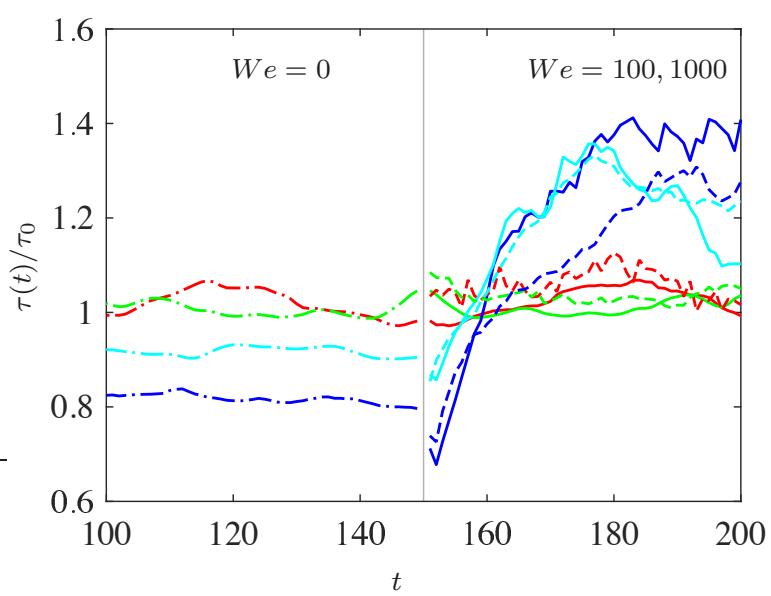

Fig. 5 (a) Wall shear stress $\tau$ normalized by the wall shear stress of the smooth wall $\tau_{0}$ : $W e=0, G F=0.5, m=0.02(-\cdot-) ; W e=0, G F=0.5, m=0.4(-.-) ; W e=0$, $G F=0.875, m=0.02(-\cdot-) ; W e=0, G F=0.875, m=0.4(-\cdot-)$. Solid lines are for $W e=1000$ and dashed for $W e=100$. The simulations at $W e>0$ are restarted from those at $W e=0$ as indicated by the vertical dotted line.

of spanwise slip, which reduces the amount of achievable drag reduction, as shown by Rastegari and Akhavan [10].

\section{Deformable interface}

A second set of simulations has been performed at Weber numbers $W e=100$ and 1,000, corresponding to some approximation to $W e^{+} \simeq 0.001$ and 0.01 respectively (Table 1 ). In such cases, the interface can deform in addition to providing a slippage. The simulations started from the last velocity and pressure field obtained at $W e=0$, to monitor how rapid is the deterioration of performances when the interface deforms. The variation in time of the wall shear stress, normalised by that of the smooth channel is shown in Figure 5 . The wall shear stress over cubes with $G F=0.875$ increases very rapidly when the Weber number is increased from 0 to 100 or 1,000. Within about 20 non-dimensional time units the drag becomes larger than that of the smooth

Table 1 Summary of the Weber number in viscous units for the different cases studied

\begin{tabular}{cccc}
\hline$m$ & $G F$ & $W e$ & $W e^{+}$ \\
\hline 0.02 & 0.5 & 1000 & $1.0168 \times 10^{-2}$ \\
0.02 & 0.875 & 1000 & $1.1592 \times 10^{-2}$ \\
0.4 & 0.5 & 1000 & $1.0135 \times 10^{-2}$ \\
0.4 & 0.875 & 1000 & $1.1205 \times 10^{-2}$ \\
0.02 & 0.5 & 100 & $1.0318 \times 10^{-3}$ \\
0.02 & 0.875 & 100 & $1.1284 \times 10^{-3}$ \\
0.4 & 0.5 & 100 & $1.0242 \times 10^{-3}$ \\
0.4 & 0.875 & 100 & $1.1319 \times 10^{-3}$ \\
\hline
\end{tabular}



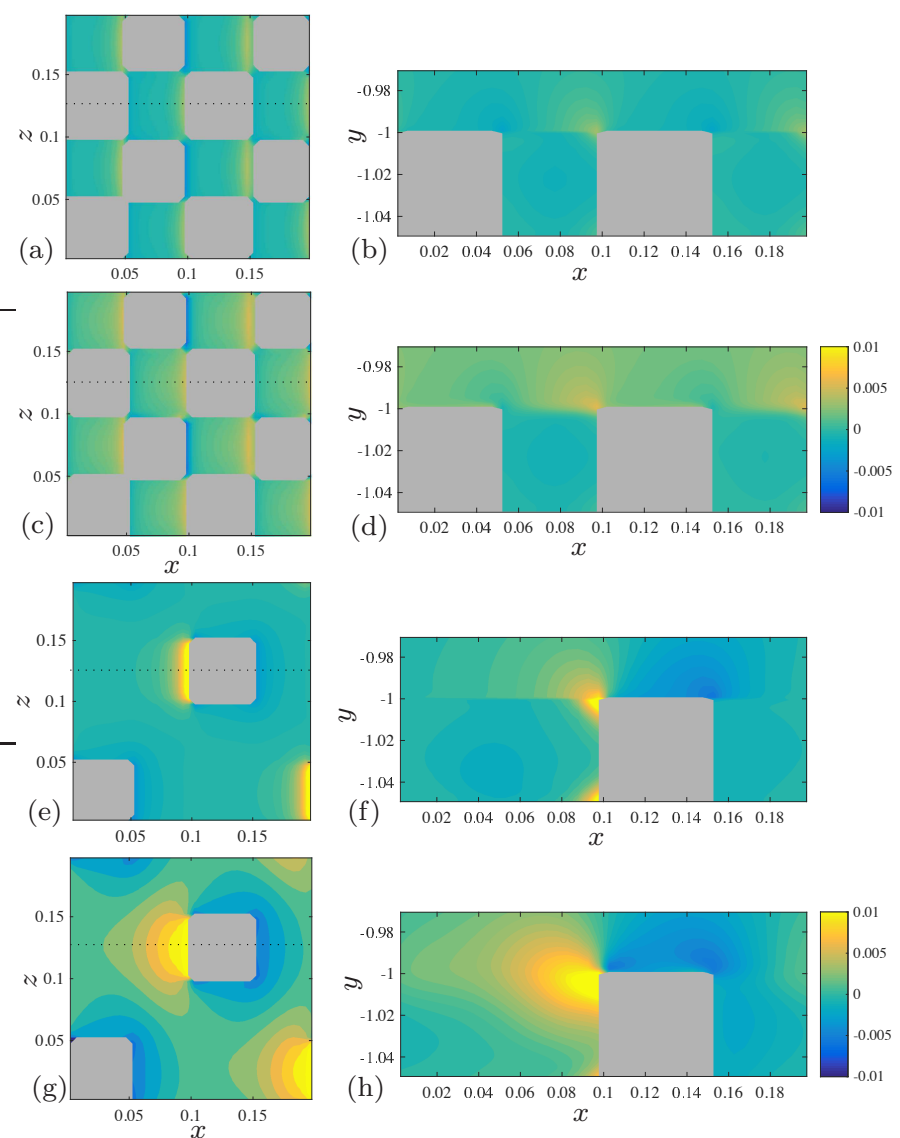

Fig. 6 Color contours of time and phase averaged pressure for $m-0.02$ : (a,b) $G F=0.5$ and $W e=0 ;(\mathrm{c}, \mathrm{d}) G F=0.5$ and $W e=1,000$. (e,f) $G F=0.875$ and $W e=0 ;(\mathrm{g}, \mathrm{h}) G F=0.875$ and $W e=1,000$.

wall. Coupling the deformation of the interface with the flow field is very important, in fact, $G F=0.875$ and $m=0.02$, which is the most effective geometry reducing the drag under the ideal condition of $W e=0$, turns out to increase the drag in more realistic conditions with a finite surface tension and a Weber number $W e=100-1,000$. Results are qualitatively similar for both Weber numbers and viscosity ratio.

For a smaller gas fraction, $G F=0.5$, the increase in drag due to the deformation of the interface is much smaller. Regardless of the Weber number, the drag remains similar to that of a smooth wall, the reduced shear at the wall being compensated by the drag inside the cavities.

The increase in drag is due to an increased pressure drag in the substrate. At $W e=0$, a stagnation point is observed at the upper corner of the cubes (Figure 6), as shown in previous studies of the flow over rough walls made of cubes. The intensity of the stagnation pressure increases with the gas fraction. 
In fact, the larger is the gas fraction, the larger is the slip velocity and therefore the pressure at the edge of the cube (scaling to some approximation to the square of the local slip velocity). For $G F=0.5$, since we consider a pinned interface, and the spacing between cubes is small, the changes in the pressure field are weak.

On the other hand, for $G F=0.875$, the distance between two cubes aligned in streamwise direction is large (i.e. $\lambda_{x}=4 k$ ) and the deformation of the interface, despite pinned to the cubes, is significant as shown in figure 7 . In spanwise direction, the larger deformation is observed in the center between two consecutive cubes. In the streamwise direction, the larger oscillation of the interface is in proximity of the leading edge of the cubes, where the stagnation pressure is larger. This is also consistent with the tendency to have ejections at the leading edge of the cubes discussed in Fig.2. The oscillation of the interface increases further the momentum transfer in the texture and as a consequence, the stagnation pressure becomes much larger than that at $W e=$ 0 . The corresponding increase of pressure drag overcomes the reduction of the shear due to the flow in the cavities, and the drag becomes larger than that over smooth walls.

Profiles of root mean square of pressure for $W e=0$ and 1,000 are shown in Figure 8. In the ideal case of a slippery interface, $(W e=0)$, the case reducing the drag the most, $G F=0.875, m=0.02$, presents pressure fluctuations smaller than those relative to the flat channel almost up to the centerline. For the other cases, in the inner part of the channel results agree well with those of [26], the effect of either LIS or SHS being weak to the outer flow. Near the crests plane, on the other hand, a large peak of pressure rms is observed. This is due to the dispersive component of the pressure, resulting from the spatial inhomogeneities in the mean pressure field shown in Fig.6. While for the ideal case of $W e=0$ the increased pressure fluctuations remain confined to a very thin layer near the interface, at $W e=1,000\left(W e^{+} \simeq 0.01\right)$ the fluctuation of pressure penetrates a larger fraction of the flow. The differences with respect to a smooth wall extend up to $0.2 h$ above the crests plane which in this case, similarly to rough walls, corresponds to 4 times the height of the cubes. Both the mean pressure as well as the fluctuating pressure are larger at $W e=1,000$. Therefore, using results from perfect slip boundary conditions may underestimate the pressure and then the deformation of the interface as also discussed in Seo et al. [16] in the context of aligned cubes.

The effect of the deformation of the interface on the drag and increased pressure fluctuations is further corroborated by visualization of iso-contours of $\lambda_{2}$ (Fig. 9). For a gas fraction $G F=0.5$, the drag and pressure fluctuations are in good approximation the same as those over a smooth channel. Consistently no significant differences are observed in the coherent structure with respect to the elongated vortices in smooth channels. On the other hand, for the drag reducing configurations, at $G F=0.875$ and $W e=0$, for both viscosity ratio, the streamwise vortices are destroyed, due to a reduced shear at the wall. As discussed previously, $G F=0.875$ is the most sensitive to the $W e$ number, and a significant deformation of the interface leads to an increased drag. The 


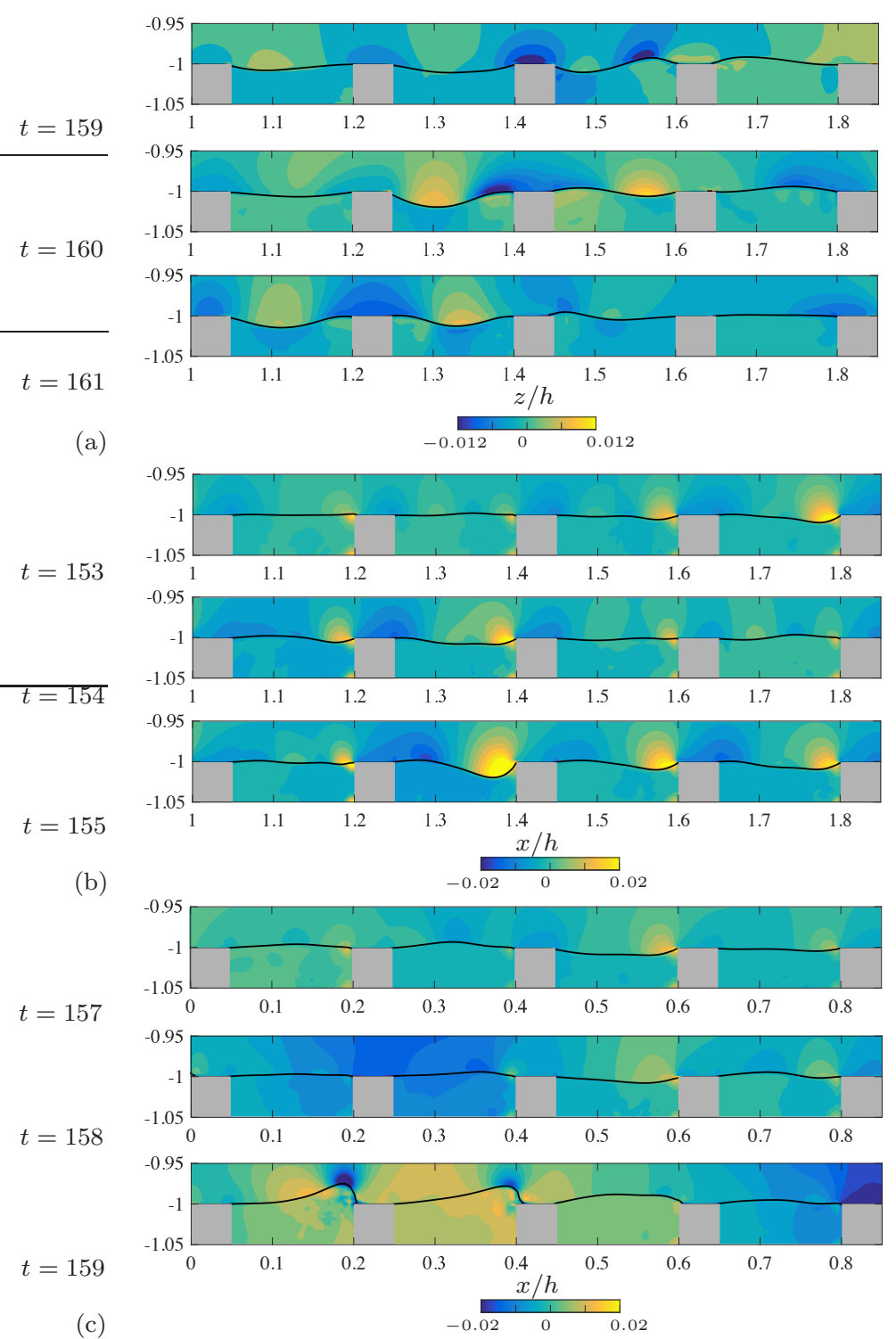

Fig. 7 Interface between the two fluids superposed to color contours of instantaneous pressure, $m=0.02, W e=1000, R e=2800, G F=0.875$ : a) vertical section in spanwise direction at $x / h=0.025$; b) vertical section in streamwise direction at $z / h=0.225$; c) vertical section in streamwise direction at $z / h=0.025$. 

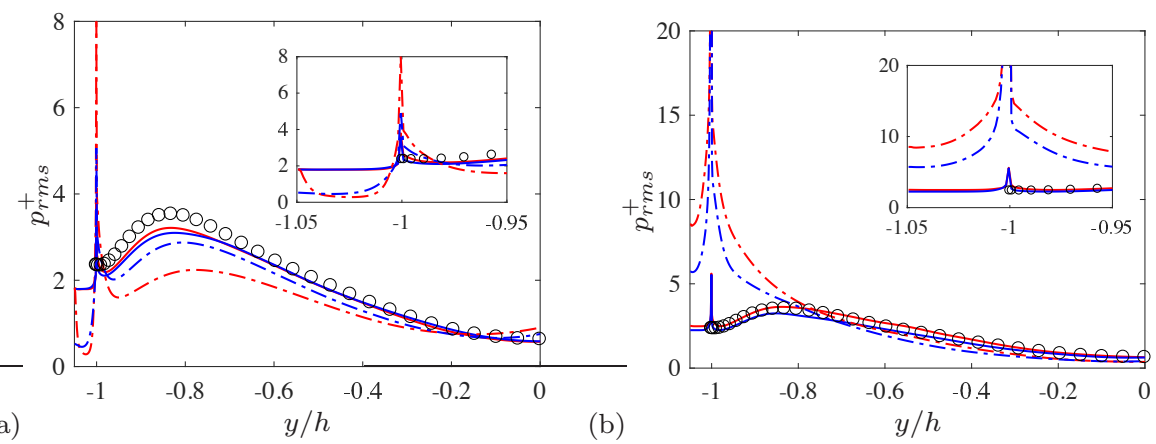

Fig. 8 Pressure root mean square at $W e=0$ (a) and $W e=1,000$ (b): $G F=0.5, m=0.02$ $(-), G F=0.5, m=0.4(-) G F=0.875, m=0.02(-\cdot-), G F=0.875, m=0.4(-\cdot-)$. DNS results from Moser et al. [26] for smooth channel with $R e_{\tau}=180(\bigcirc)$ are included as reference.

iso-contours of $\lambda_{2}$ consistently are more intense than those over a smooth wall resembling more closely those relative to rough walls (Leonardi et al. [27]).

\section{Conclusion}

Direct Numerical Simulations of two superposed fluids in a channel have been performed. One of the fluids is locked into a textured surface made of staggered cubes on the lower wall of the channel. Two gas fractions $(G F=0.5$ and 0.875 ) have been considered. A set of simulations has been carried out considering the case of a flat and slippery interface to the streamwise and spanwise velocity but not deformable in the wall normal direction. This corresponds to an ideal case of an infinite surface tension (and then $W e=0$ ) which damps any deformation induced by the stresses normal to the interface. Results agree well with the analytical models of $[10,9,23]$ despite those were obtained with Stokes approximation and we do not neglect the drag in the textured surfaces. Considering the mean flow patterns, we defined an equivalent pitch for staggered cubes to use Ybert's model for aligned cubes.

A second set of simulations, with a more realistic value of surface tension, and Weber numbers $W e=100$ and 1,000 corresponding to some approximation to $W e^{+} \simeq 10^{-3}$ and $10^{-2}$ has been performed. The dynamics of the interface is coupled with the Navier-Stokes equations, and the interface is pinned to the cubes. The simulations were started from a velocity field obtained for the same gas fraction and viscosity ratio at $W e=0$. The degradation of performances in terms of drag reduction are rather quick, and for a $G F=0.875$ the surface transitions from being drag reducing (about 20\%) to drag increasing (about $40 \%$ ) within 20 non-dimensional time units. The drag does not increase because the interface breaks and there is a transition from Cassie to Wenzel state. In fact, the interface in the present simulations is pinned to the cubes. We discovered that the dynamics of the interface alone, even when pinned to the textured surface may increase the turbulence production and the drag. 

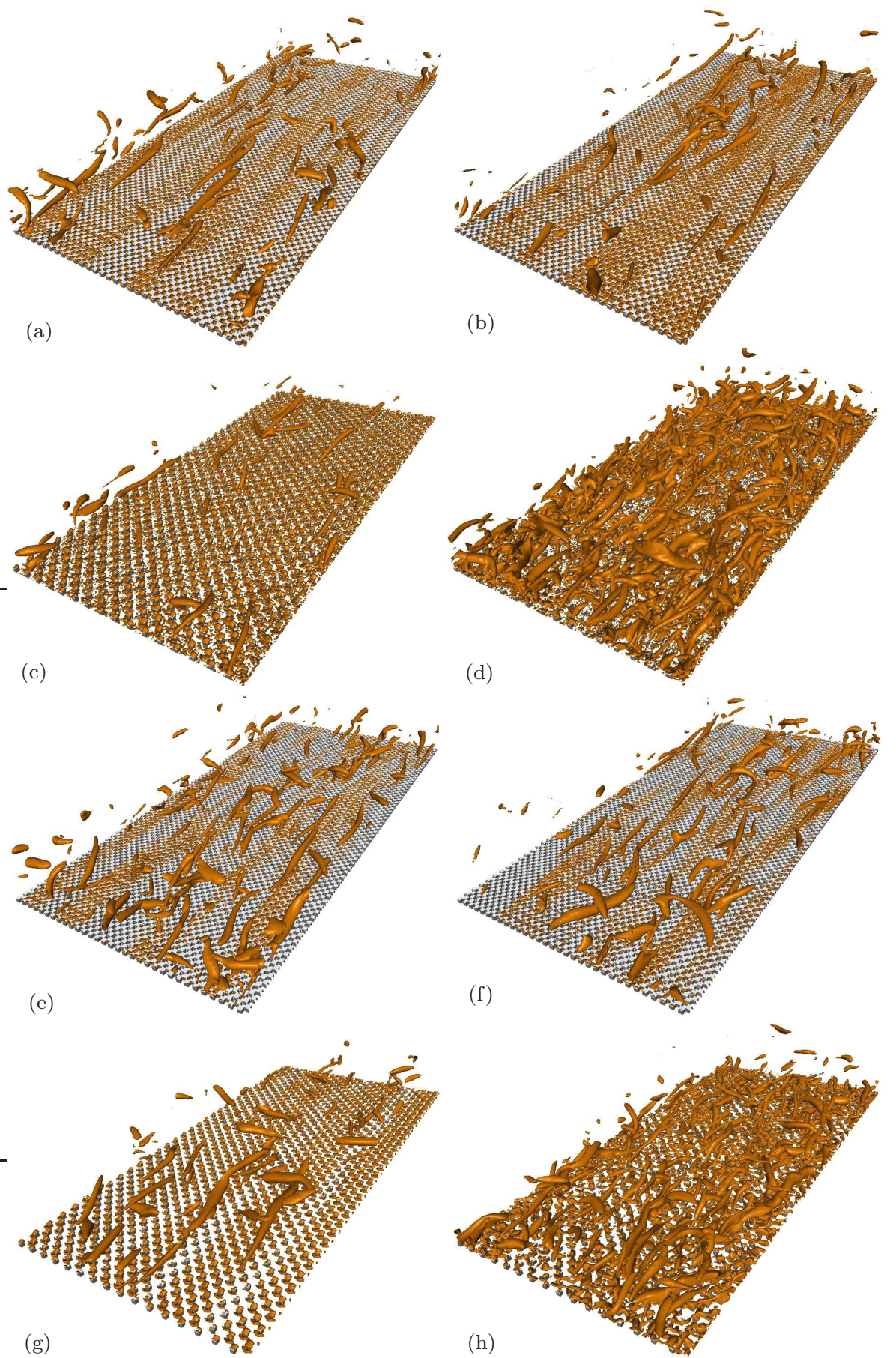

Fig. 9 Iso-contours of $\lambda_{2}$ criterion for SHSs $(m=0.02$ ). (a) $G F=0.5 W e=0$, (b) $G F=0.5$ $W e=1000$, (c) $G F=0.875 W e=0,(\mathrm{~d}) G F=0.875 W e=1000 ;$ LISs $(m=0.4)$. (e) $G F=0.5 W e=0,(f) G F=0.5 W e=1000,(\mathrm{~g}) G F=0.875 W e=0,(\mathrm{~h}) G F=0.875$ $W e=1000$. 
Therefore, it is not sufficient that the surface remains in the Cassie state to have drag reduction, but the deformation of the interface needs to be small enough. The detrimental effect on the drag due of the deformation of the interface increases with the gas fraction. In fact, present results show that, for a quite dense substrate $(G F=0.5)$, the drag is almost unaffected by the Weber number or in other words by the deformation of the interface.

While simulations and analytical derivation based on perfect slip conditions $(W e=0)$ predict very high value of drag reduction when the gas fraction is large, present results seem to indicate that high gas fraction will actually increase the drag due to the detrimental effect of the dynamics of the interface. The gas fraction, on one hand, increases the theoretical drag reduction, by allowing a larger slip length (or velocity). On the other hand, the deformation of the interface, increases with the gas fraction, leading to an increase of drag. Present results suggest that the gas fraction should be the results of an optimization process balancing the effects of an increased slip length with that of an increased deformation of the interface. We also point out that the present results have been obtained for staggered micro-posts and cannot be directly extrapolated to different geometrical patterns. Staggered microposts are known to develop very large stagnation pressure upon impact of the flow to the back of the posts and are characterized by a reduced stability of the interaface in comparison with aligned micro-posts and longitudinal micro-grooves.

\section{Compliance with Ethical Standards:}

Funding: Stefano Leonardi, Isnardo Arenas and Edgardo Garcia were supported under ONR MURI Grants N00014-12-0875 and N00014-12-1-0962. The authors declare that they have no conflict of interest.

\section{References}

1. B.J. Rosenberg, T. Van Buren, M.K. Fu, A.J. Smits, Physics of Fluids 28(1) (2016)

2. T. Van Buren, A. Smits, Journal of Fluid Mechanics 827, 448 (2017)

3. J.S. Wexler, A. Grosskopf, M. Chow, Y. Fan, I. Jacobi, H.A. Stone, Soft Matter 11(25), $5023(2015)$

4. M.B. Martell, J.B. Perot, J.P. Rothstein, Journal of Fluid Mechanics 620, 31 (2009)

5. M.B. Martell, J.P. Rothstein, J.B. Perot, Physics of Fluids 22(6), 1 (2010)

6. H. Park, H. Park, J. Kim, Physics of Fluids 25(11) (2013)

7. S. Türk, G. Daschiel, A. Stroh, Y. Hasegawa, B. Frohnapfel, Journal of Fluid Mechanics 747, $186217(2014)$

8. M.K. Fu, I. Arenas, S. Leonardi, M. Hultmark, Journal of Fluid Mechanics 824, 688 (2017)

9. C. Schönecker, T. Baier, S. Hardt, Journal of Fluid Mechanics 740, 168 (2014)

10. A. Rastegari, R. Akhavan, Journal of Fluid Mechanics 773, R4 (2015)

11. E. Aljallis, M.A. Sarshar, R. Datla, V. Sikka, A. Jones, C.H. Choi, Physics of Fluids 25(2) (2013)

12. J.S. Wexler, I. Jacobi, H.A. Stone, Physical Review Letters 114(16), 1 (2015) 
13. K.B. Golovin, J.W. Gose, M. Perlin, S.L. Ceccio, A. Tuteja, Philosophical Transactions of the Royal Society A: Mathematical, Physical and Engineering Sciences 374(2073), 20160189 (2016)

14. A.P. Hooper, W.G.C. Boyd, Journal of Fluid Mechanics 179, 201 (1987)

15. E.J. Hinch, Journal of Fluid Mechanics 144, 463 (1984)

16. J. Seo, R. García-Mayoral, A. Mani, Journal of Fluid Mechanics 783, 448 (2015)

17. P. Orlandi, Fluid Flow Phenomena: A Numerical Toolkit, vol. 55 (Springer, 2000)

18. P. Orlandi, S. Leonardi, Journal of Turbulence 7, N73 (2006)

19. J.A. Sethian, P. Smereka, Annual Review of Fluid Mechanics 35(1), 341 (2003)

20. S. Osher, R.P. Fedkiw, Journal of Computational Physics 169(2), 463 (2001)

21. G. Russo, P. Smereka, Journal of Computational Physics 163(1), 51 (2000)

22. Y. Renardy, M. Renardy, Journal of Computational Physics 183(2), 400 (2002)

23. C. Ybert, C. Barentin, C. Cottin-Bizonne, P. Joseph, L. Bocquet, Physics of Fluids 19(12) (2007)

24. P. Luchini, F. Manzo, A. Pozzi, Journal of Fluid Mechanics 228, 87 (1991)

25. E. Lauga, H.A. Stone, Journal of Fluid Mechanics 489, 5577 (2003)

26. R.D. Moser, J. Kim, N. Mansour, Physics of Fluids 11, 943 (1999)

27. S. Leonardi, P. Orlandi, L. Djenidi, R. Antonia, International Journal of Heat and Fluid Flow 25(3), 384 (2004). Turbulence and Shear Flow Phenomena (TSFP-3) 